\title{
Distúrbios no Perfil Lipídico São Altamente Prevalentes em População Nipo-Brasileira
}

\section{artigo original}

\author{
Antonela F. A. Siqueira \\ Helena A. Harima \\ Katsumi Osiro \\ AMÉLIA T. HIRAI \\ Suely G. A. GIMENo \\ SANDRA R. G. FERREIRA \\ JAPANESE-BRAZIIIAN DIABETES \\ StUdY Group
}

Departamento de Medicina da Universidade Federal de São Paulo (Unifesp/EPM), São Paulo, SP Brasil (AFAS); Departamento de Medicina Preventiva da Unifesp/EPM, São Paulo, SP, Brasil (HAH, KO, ATH, SGAG);

Departamento de Nutrição da Faculdade de Saúde Pública da Universidade de São Paulo (USP), SP, Brasil (SRGF); JapaneseBrazilian Diabetes Study Group (JBDSG) (ver apêndice)

Recebido em 21/03/2007 Aceito em 21/09/2007
RESUMO

Alta prevalência de diabetes em população nipo-brasileira de Bauru/SP foi previamente relatada. Visando a complementar a avaliação do risco cardiometabólico, este estudo analisou distúrbios no perfil lipídico de 1.330 nipobrasileiros (46\% homens) $>30$ anos. Definiu-se hipercolesterolemia por níveis de colesterol total $\geq 240 \mathrm{mg} / \mathrm{dL}$; hipertrigliceridemia por valores $\geq 150 \mathrm{mg} / \mathrm{dL}$ e HDL-colesterol baixo por valores $<40 \mathrm{mg} / \mathrm{dL}$ e $<50 \mathrm{mg} / \mathrm{dL}$ para homens e mulheres, respectivamente. A prevalência desses distúrbios foi comparada pelo qui-quadrado, estratificando-se por sexos e categorias glicêmicas. As médias dos lipídeos e lipoproteínas foram comparadas entre sexos pelo teste $t$ de Student. A prevalência de hipertrigliceridemia foi de 66,0\% (IC95\%:63,5 $-68,5 \%$ ), sendo mais comum em homens e aumentando com a piora da categoria glicêmica; a trigliceridemia média foi $235,7 \pm 196,3 \mathrm{mg} / \mathrm{dL}$. A prevalência de hipercolesterolemia foi 24,4\% (IC95\%:22,1 - 26,7\%); HDL-C baixo foi observado em 43,0\% (IC95\%:39,4 - 46,6\%] das mulheres e 17,5\% (IC95\%:14,5-20,5\%) dos homens, porém a razão colesterol total/HDL-C foi menor em mulheres $(4,23 \pm 0,68$ vs. 4,40 $\pm 0,73 ; p<0,001)$. Em nipo-brasileiros, a hipertrigliceridemia é a anormalidade lipídica mais comum, em concordância com a elevada prevalência de diabetes. Os homens apresentaram pior perfil lipídico que as mulheres. Sugere-se que hábitos de vida ocidental possam estar deteriorando a saúde desses indivíduos. (Arq Bras Endocrinol Metab 2008; 52/1:40-46)

Descritores: Dislipidemias; Nipo-brasileiros; Diabetes; Risco cardiometabólico

\section{ABSTRACT}

Lipid Profile Disturbances Are Highly Prevalent in Japanese-Brazilians. High prevalence of diabetes has been previously reported in Japanese-Brazilians. In an attempt to better estimate the cardiometabolic risk, this study evaluated lipid disorders in 1,330 Japanese-Brazilians (46\% men) aged $>30$ years. Hypercholesterolemia was defined as serum cholesterol $\geq 240 \mathrm{mg} / \mathrm{dL}$, hypertriglyceridemia as values $\geq 150 \mathrm{mg} / \mathrm{dL}$ and low-HDL-C as values $<40 \mathrm{mg} / \mathrm{dL}$ and $<50 \mathrm{mg} / \mathrm{dl}$ for men and women respectively. The prevalence of dyslipidemias was compared by the chi-square test between gender and glycemic category. Mean and Standard Deviation of lipids and lipoproteins were compared by the Student t-Test between gender. Hypertriglyceridemia was detected in $66.0 \%$ [95\% Cl: $63.5-68.5]$ of the population, being more common in men and increasing with deterioration of glucose metabolism. Mean level of triglycerides was $235.7 \pm 196.3 \mathrm{mg} / \mathrm{dL}$. The prevalence of hypercholesterolemia was $24.4 \%$ [ $95 \%$ Cl: 22.1- 26.7]. Low HDL-C was observed in 17.5\% [95\% Cl: 14.5-20.5] of men and $43.0 \%$ [95\% Cl: 39.4- 46.6] of women but total / HDL-cholesterol ratio was lower in women $(4.23 \pm 0.68$ vs. $4.40 \pm 0.73, p<0.001)$. In Japanese-Brazilians, hypertriglyceridemia is the commonest dyslipidemia, in agreement with the high prevalence of diabetes. Men showed a worse lipid profile than women; it 
was suggested that the Western diet and living habits could be deteriorating their health. (Arq Bras Endocrinol Metab 2008;52/1:40-46)

Keywords: Dyslipidemias; Japanese-Brazilians; Diabetes; Cardiometabolic risk

\section{INTRODUÇÃO}

$\mathrm{D}$ IVERSOS ESTUDOS CONDUZIDOS em migrantes japoneses nas Américas detectaram alta prevalência de diabetes melito tipo 2 (DM2) nesses indivíduos $(1,2)$. A população nipo-brasileira residente em Bauru/SP, apresenta uma das maiores taxas registradas no mundo (1); tais indivíduos possuem elevado risco cardiovascular. De fato, estudos prévios do nosso grupo demonstraram que $14 \%$ da população apresentou alguma manifestação de doença macrovascular (3).

É consagrado que a hipercolesterolemia isolada contribui de forma significante para a formação da placa aterosclerótica, e em vigência da resistência à insulina (RI), o aumento do LDL-colesterol (LDL-C) torna-se ainda mais nocivo, em decorrência das modificações que ocorrem na lipoproteína de baixa densidade (LDL), como oxidação e glicação, aumentando seu potencial aterogênico (4). Nessa situação clínica, observa-se a presença de LDLs pequenas e densas, com níveis elevados de apolipoproteína B (proteína estrutural das LDLs e VLDLs), elevação dos triglicérides (TGs) e baixa concentração da lipoproteína de alta densidade (HDL), partícula que poderia atuar no transporte reverso do colesterol, reduzindo a velocidade de formação da placa (5). Com base nessas alterações lipídicas, típicas da síndrome metabólica (SM), o National Cholesterol Education Program - Adult Treatment Panel III (NCEP ATP III) sugere alvos mais rigorosos para a LDL, à medida que se adicionam fatores de risco cardiovascular, principalmente na presença de DM2 (6).

Durante a última década, a população japonesa residente em seu país de origem vem mostrando aumento em suas taxas de colesterol e triglicérides (7). O Brasil conta com a maior população de origem japonesa residente fora de seu país de origem (8), representando importante modelo epidemiológico para avaliar o impacto dos hábitos de vida ocidentais na saúde desses indivíduos, geneticamente japoneses.

Em nosso meio, dados populacionais sobre prevalência de dislipidemias são escassos. Considerando-se que vivenciamos uma epidemia global de obesidade e DM2 (9), marcadamente no Ocidente, é de grande interesse aprofundar-se no estudo do perfil lipídico de nossa população, em particular de um estrato populacional - descendentes japoneses -, cujas condições metabólicas mostram-se incontestavelmente afetadas pelo ambiente ocidental (10).

Este estudo se propõe a avaliar a prevalência das dislipidemias - hipercolesterolemia, hipertrigliceridemia e HDL-colesterol (HDL-C) baixo - numa populaçãonipo-brasileira, fornecendo dados para comparação com estudos de amostras brasileiras e do Japão. Tal conhecimento permitirá aprofundar a avaliação do risco cardiovascular global desses indivíduos e orientar medidas de prevenção.

\section{MÉTODOS}

O Japanese-Brazilian Diabetes Study Group (JBDSG) conduziu uma pesquisa em indivíduos de origem japonesa não-miscigenada, residente no município de Bauru, SP, para determinar a prevalência de diabetes e doenças associadas nessa população (1). O estudo foi aprovado pelo comitê de ética da Universidade Federal de São Paulo, tendo os participantes assinado termo de consentimento. No ano de 2000, foram avaliados 1.330 nipobrasileiros isseis (nascidos no Japão) ou niseis (filhos de isseis, nascidos no Brasil) de ambos os sexos, acima de 30 anos de idade. Os detalhes da seleção e do recrutamento da população foram previamente reportados (1).

Dados de história clínica foram obtidos por questionário padronizado; os participantes foram submetidos a exame médico e coletas de sangue em jejum (12 horas); aqueles indivíduos que não referiam diabetes, com glicemia capilar $<200 \mathrm{mg} / \mathrm{dL}$, foram submetidos ao teste oral de tolerância à glicose. As lipoproteínas séricas e os TGs foram dosados por método enzimáti$\mathrm{co}$, e a glicemia pela glicose-oxidase. Posteriormente, em soro congelado a $-70{ }^{\circ} \mathrm{C}$ de uma amostra randomizada de 362 indivíduos com características demográfi- 
cas semelhantes a da população total, foi dosada a apolipoproteína B (apoB) por ensaio imunoturbidimétrico (faixa de normalidade: 66 a $133 \mathrm{mg} / \mathrm{dL}$ para homens e 60 a $117 \mathrm{mg} / \mathrm{dL}$ para mulheres).

O diagnóstico de hipercolesterolemia foi feito quando as concentrações séricas de colesterol total eram $\geq 240 \mathrm{mg} / \mathrm{dL}$ e o de hipertrigliceridemia quando os níveis de TG eram $\geq 150 \mathrm{mg} / \mathrm{dL}$. HDL-C baixo foi diagnosticado por níveis de HDL $<40 \mathrm{mg} / \mathrm{dL}$ para homens e $<50 \mathrm{mg} / \mathrm{dL}$ para mulheres (6). O uso estatinas ou fibratos não foi utilizado como parâmetro diagnóstico em virtude da subutilização destes pelos nipo-brasileiros - apenas 22 indivíduos na população total estavam em tratamento com tais medicações. A inclusão desses indivíduos no grupo dislipidêmico não alterou os resultados finais obtidos.

\section{Análise estatística}

As taxas de prevalência foram calculadas por ponto e intervalo com $95 \%$ de confiança e comparadas entre sexos pelo teste do qui-quadrado. Foi calculada a razão de prevalência de hipertrigliceridemia entre indivíduos com e sem DM2. As variáveis demográficas, clínicas e bioquímicas foram expressas em médias e desvios-padrão e comparadas pelo teste $t$ de Student. Correlações da idade com os níveis séricos dos lipídeos e lipoproteínas, assim como dos TGs com glicemia de jejum e duas horas após sobrecarga, cintura, índice de massa corporal (IMC) e HOMA-R, foram realizadas pelo coeficiente de Pearson. Para variáveis não-paramétricas (TG e HDL) foi empregada transformação logarítmica antes da análise.

\section{RESULTADOS}

Entre os 1.330 indivíduos, $613(46,1 \%)$ eram do sexo masculino, e a idade média da população foi $57,0 \pm$ 12,4 anos, não diferindo entre homens e mulheres $(57,0$ $\pm 12,8$ vs. $56,9 \pm 12,2$ anos, respectivamente). O IMC foi maior no sexo masculino $\left(25,3 \pm 3,8 \mathrm{~kg} / \mathrm{m}^{2} v s .24,6\right.$ $\left.\pm 3,9 \mathrm{~kg} / \mathrm{m}^{2} ; \mathrm{p}<0,01\right)$, assim como a circunferência da cintura $(88,4 \pm 9,6 \mathrm{~cm}$ vs. $80,0 \pm 9,5 \mathrm{~cm} ; \mathrm{p}<0,001)$, a pressão arterial média $(99,2 \pm 15,5 \mathrm{mmHg}$ vs. $95,1 \pm 16,2$ mmHg; $\mathrm{p}<0,001)$ e a glicemia de jejum $(128,0 \pm 33,9$ $\mathrm{mg} / \mathrm{dL}$ vs. $122,4 \pm 35,1 \mathrm{mg} / \mathrm{dL} ; \mathrm{p}<0,01)$. Os valores de glicemia duas horas após sobrecarga foram maiores nos homens, porém sem significância estatística (171,3 \pm 84,9 $\mathrm{mg} / \mathrm{dL} v s .163,3 \pm 71,8 \mathrm{mg} / \mathrm{dL} ; \mathrm{p}>0,05)$.

As prevalências das alterações do perfil lipídico na população total e por sexos encontram-se na tabela 1 . As mulheres apresentam maior prevalência de hipercolesterolemia e HDL-C baixo que os homens; no entanto, no sexo masculino, a hipertrigliceridemia é mais prevalente que no feminino.

Na tabela 2 encontram-se as prevalências das alterações do perfil lipídico de acordo com o grau de tolerância à glicose. A razão das prevalências de hipertrigliceridemia dos indivíduos com e sem DM2 foi de 1,35.

Foram detectadas correlações significantes dos valores de TGs com a glicemia de jejum $(r=0,27 ; \mathrm{p}<0,001)$ e a glicemia pós-sobrecarga $(\mathrm{r}=0,25 ; \mathrm{p}<0,001)$. Os TGs também se correlacionaram com as medidas de cintura $(\mathrm{r}=0,34 ; \mathrm{p}<0,001)$, IMC $(\mathrm{r}=0,28 ; \mathrm{p}<0,001) \mathrm{e}$ com o HOMA-R $(r=0,22 ; \mathrm{p}<0,001)$.

A tabela 3 mostra valores séricos médios dos lipídeos, lipoproteínas e apoB na população total e comparadas pelo sexo. A média do LDL-C foi maior nas mulheres, porém, as médias da razão colesterol total/HDL-C e dos TGs foram mais elevadas nos homens. A média do HDL-C foi maior no sexo feminino. Não se observou diferença significante nos níveis de apoB entre os sexos.

A figura 1 mostra a distribuição populacional nas faixas do LDL-C, propostas como alvos terapêuticos pelo NCEP - ATP III (6). Observa-se que um quinto da população nipo-brasileira apresentou níveis de LDL$\mathrm{C}<100 \mathrm{mg} / \mathrm{dL}$ e que aproximadamente a metade dos indivíduos apresentou níveis $>130 \mathrm{mg} / \mathrm{dL}$.

Tabela 1. Prevalência das dislipidemias em população nipo-brasileira, estratificada pelo sexo.

\begin{tabular}{lccc}
\hline & $\begin{array}{c}\text { Homens } \\
\text { \% (IC 95\%) }\end{array}$ & $\begin{array}{c}\text { Mulheres } \\
\% \text { (IC 95\%) }\end{array}$ & $\begin{array}{c}\text { Total } \\
\%(\text { IC 95\%) }\end{array}$ \\
\hline Hipercolesterolemia & $21,9(18,6-25,2)$ & $26,5(23,3-29,7)^{*}$ & $24,4(22,1-26,7)$ \\
\hline HDL-C baixo & $17,5(14,5-20,5)$ & $43,0(39,4-46,6)^{\#}$ & $31,2(28,7-33,7)$ \\
\hline Hipertrigliceridemia & $73,6(70,1-77,1)$ & $59,6(56,0-63,2)^{\#}$ & $66,0(63,5-68,5)$ \\
\hline
\end{tabular}

${ }^{*} \mathrm{p}<0,050$ versus homens; $\quad \mathrm{p}<0,001$ versus homens. 
Tabela 2. Prevalência das dislipidemias em população nipo-brasileira, estratificada pelas categorias de tolerância à glicose.

\begin{tabular}{|c|c|c|c|c|c|}
\hline & $\begin{array}{c}\text { Normais } \\
(n=69) \\
\%(I C 95 \%)\end{array}$ & $\begin{array}{c}\text { GJA } \\
(n=467) \\
\%(\operatorname{IC~} 95 \%)\end{array}$ & $\begin{array}{c}\text { TGD } \\
(n=317) \\
\%(I C 95 \%)\end{array}$ & $\begin{array}{c}\text { Diabetes tipo } 2 \\
(n=477) \\
\%(I C 95 \%)\end{array}$ & $\begin{array}{c}\text { Total } \\
(n=1330) \\
\%(\text { IC } 95 \%)\end{array}$ \\
\hline Hipercolesterolemia & $10,1(3,0-17,2)$ & $22,7(18,9-26,5)^{*}$ & $27,4(22,5-32,3)^{\#}$ & $26,0(22,1-29,9)^{\#}$ & $24,4(22,1-26,7)$ \\
\hline HDL-C baixo & $36,2(24,9-47,5)$ & $29,6(25,5-33,7)$ & $32,8(27,6-38,0)$ & $31(26,8-35,1)$ & $31,2(28,7-33,7)$ \\
\hline Hipertrigliceridemia & $34,8(23,6-46,0)$ & $54,2(49,7-58,7)^{*}$ & $71,9(66,9-76,8)^{\#+}$ & $78,2(74,5-81,9)^{\#+}$ & $66,0(63,5-68,5)$ \\
\hline $\begin{array}{l}G J A=\text { glicemia de jeju } \\
T G D=\text { tolerância a glic }\end{array}$ & $\begin{array}{l}\text { terada } \\
\text { diminuída }\end{array}$ & $\begin{array}{l}{ }^{*} \mathrm{p}<0,05 \text { versus } \mathrm{no} \\
\# \mathrm{p}<0,001 \text { versus } \mathrm{n}\end{array}$ & $\begin{array}{l}\text { mais } \\
\text { prmais }\end{array}$ &, 05 versus GJA & \\
\hline
\end{tabular}

Tabela 3. Lipides e lipoproteínas séricas em população nipo-brasileira, estratificados pelo sexo.

\begin{tabular}{lccc}
\hline & $\begin{array}{c}\text { Homens } \\
(\mu \pm \mathrm{DP})\end{array}$ & $\begin{array}{c}\text { Mulheres } \\
(\mu \pm \mathrm{DP})\end{array}$ & $\begin{array}{c}\text { Total } \\
(\mu \pm \mathrm{DP})\end{array}$ \\
Colesterol total $(\mathrm{mg} / \mathrm{dL})$ & $212,7 \pm 42,3$ & $216,2 \pm 42,0$ & $214,6 \pm 42,2$ \\
Colesterol LDL $(\mathrm{mg} / \mathrm{dL})$ & $127,9 \pm 37,8$ & $132,2 \pm 38,2^{*}$ & $130,2 \pm 38,0$ \\
Colesterol HDL (mg/dL) & $49,3 \pm 12,3$ & $51,8 \pm 10,1^{\#}$ & $50,6 \pm 11,2$ \\
Razão colesterol total / HDL & $4,40 \pm 0,73$ & $4,23 \pm 0,68^{\#}$ & $4,31 \pm 0,71$ \\
Triglicérides (mg/dL) & $270,7 \pm 230,2$ & $205,9 \pm 155,8^{\#}$ & $235,7 \pm 196,3$ \\
Apolipoproteína B (mg/dL) & $93,2 \pm 25,3$ & $89,5 \pm 28,5$ & $91,2 \pm 27,0$ \\
\hline
\end{tabular}

" $\mathrm{p}<0,050$ versus homens. $\quad \mathrm{p}<0,001$ versus homens. Resultados expressos em média \pm desvio-padrão.

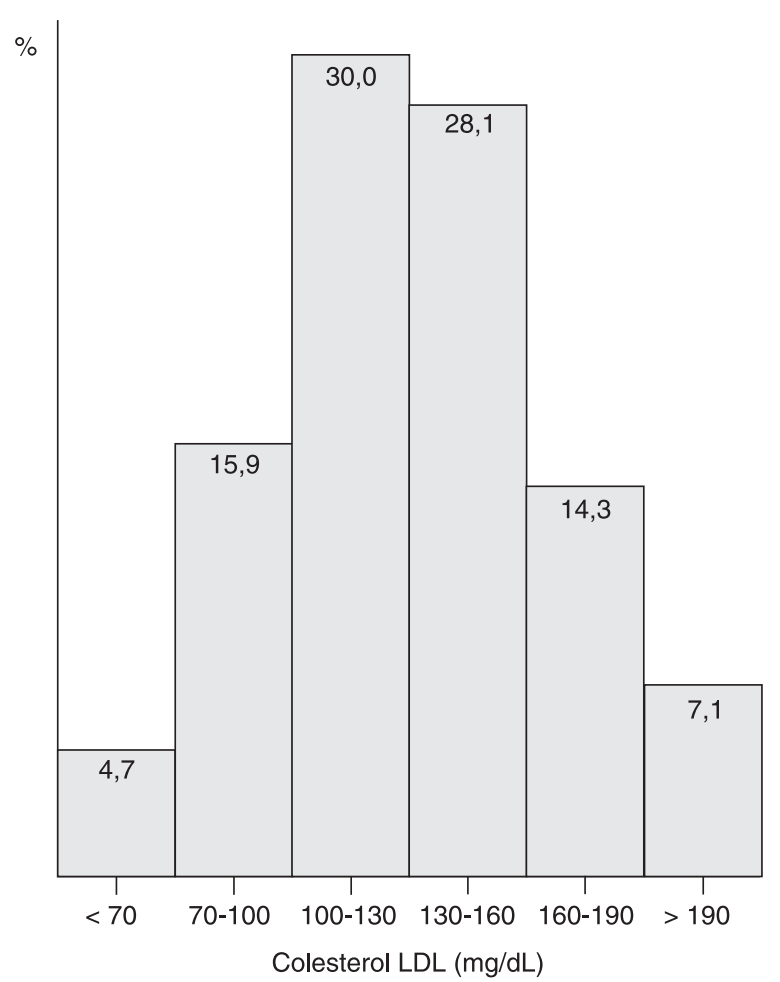

Figura 1. Distribuição populacional do colesterol LDL em nipo-brasileiros.
Foram detectadas correlações significativas, porém fracas da idade com o colesterol total $(\mathrm{r}=0,11 ; \mathrm{p}<$ $0,001)$, assim como com o LDL-C e o HDL-C ( $\mathrm{r}=$ 0,$10 ; \mathrm{p}<0,001$ e $\mathrm{r}=0,07 ; \mathrm{p}<0,050$, respectivamente); entretanto, não houve correlação da idade com os níveis de TGs ou de apoB.

\section{DISCUSSÃo}

A dislipidemia mais freqüentemente encontrada nos nipo-brasileiros foi a hipertrigliceridemia (66\% apresentaram valores $\geq 150 \mathrm{mg} / \mathrm{dL}$ ), o que não surpreende diante da alta prevalência de DM2 (35\%) já relatada nessa população (1). O controle glicêmico inadequado (apenas 5,3\% dos indivíduos apresentaram glicemia de jejum e pós-sobrecarga dentro dos limites da normalidade) e a presença de RI devem explicar em parte a elevada prevalência de hipertrigliceridemia. Esse fato é reforçado pela associação dos TGs com as glicemias de jejum e póssobrecarga e com indicativos de RI (circunferência da cintura e HOMA-IR) detectados na presente análise, e também pelo incremento observado na prevalência de hipertrigliceridemia conforme se deteriora o perfil glicí- 
dico desses indivíduos. Outro trabalho do nosso grupo observou associação entre o consumo elevado de frutas, sucos de frutas, pão branco e arroz refinado com a intolerância à glicose (11), mas a hipótese de que esses alimentos poderiam aumentar os níveis de TGs ainda não foi testada nessa população.

Comparando nossa população com a norte-americana do Estudo Multi-Ethnic Study of Atherosclerosis (MESA) (12), no qual foram avaliados mais de 6.000 indivíduos entre 45 e 84 anos, sem diagnóstico prévio de doença cardiovascular, nota-se que as concentrações séricas dos TGs foram consideravelmente maiores nos nipo-brasilei$\operatorname{ros}(235,7 \mathrm{mg} / \mathrm{dL}$ vs. $125,9 \mathrm{mg} / \mathrm{dL})$. Esse fato pode ser decorrente da menor prevalência de DM2 observada na população do MESA (15\%) e também da maior utilização de medicações por esses indivíduos - mais da metade dos participantes com dislipidemia estavam em uso de hipolipemiantes, correspondendo a $16 \%$ da população total, ao passo que nos nipo-brasileiros o emprego desses medicamentos foi insignificante.

Na série brasileira do município de Cotia/SP (13), onde foram incluídos indivíduos acima de 20 anos de idade, os níveis de TGs também se mostraram inferiores aos da população nipo-brasileira (TGs $=110,9 \mathrm{mg} / \mathrm{dL}$ em mulheres e $124,5 \mathrm{mg} / \mathrm{dL}$ em homens). No Japão, um estudo avaliou 13.000 indivíduos dos 4 aos 99 anos, com níveis glicêmicos mais baixos (glicemia de jejum = $95 \mathrm{mg} / \mathrm{dL}$ ) (7). Mesmo comparada aos nipobrasileiros sem alteração no metabolismo glicídico, a trigliceridemia média foi menor nos japoneses vivendo em seu país de origem (118 mg/dL vs. 140,5 mg/dL nos nipo-brasileiros - dados não mostrados). Esses achados reforçam a hipótese do JBDSG de que indivíduos de origem japonesa, expostos ao ambiente ocidental, são altamente predispostos a distúrbios metabólicos relacionados à $\mathrm{RI}$ - como a hipertrigliceridemia e o DM2 - que podem decorrer da tendência à deposição abdominal de gordura corporal (14).

O valor médio do HDL-C nos nipo-brasileiros $(50,6 \mathrm{mg} / \mathrm{dL})$, apesar de menor que o dos japoneses vivendo em seu país de origem (7) (59 mg/dL), assemelhou-se ao encontrado na população do MESA (12) $(51,2 \mathrm{mg} / \mathrm{dL})$ e foi discretamente maior que a do município de Cotia (13) (46 mg/dL em mulheres e 43,5 $\mathrm{mg} / \mathrm{dL}$ em homens). As razões para o HDL-C dos nipo-brasileiros serem menos afetados que os TG pelo ambiente ocidental não são claras, especialmente conhecendo o baixo nível de atividade física dessa população em geral (15).
Ao se analisar os distúrbios metabólicos separadamente por sexo, nota-se que os homens são mais afetados pela hipertrigliceridemia e também pelo DM2 (1) do que as mulheres. Em análise prévia, o consumo de bebidas alcoólicas foi maior nos nipo-brasileiros do sexo masculino (16), podendo contribuir para a maior prevalência dessa dislipidemia nos homens. No que se refere à ocorrência de HDL-C baixo, essa anormalidade foi mais prevalente nas mulheres; no entanto, cabe ressaltar que o critério do NCEP para definir HDL-C baixo, como parâmetro diagnóstico de $\mathrm{SM}$, é mais rigoroso para o sexo feminino (6). Por outro lado, com base em análise prévia nessa população que mostrou que as mulheres eram menos ativas que os homens (15), há especulações se esse fato poderia estar associado à maior prevalência de $\mathrm{HDL}$ baixo nesse sexo. Ainda assim, o sexo masculino apresentou valores médios do HDL-C menores e também maior razão colesterol total/HDL, o que juntamente à maior prevalência de DM2, coloca os homens sob condição de maior risco cardiovascular do que as mulheres.

Na presente análise, observou-se também que a hipercolesterolemia é bastante comum nos nipo-brasileiros, afetando cerca de um quarto dos indivíduos. Esses números assemelham-se aos encontrados em 7.392 adultos que tiveram seu perfil lipídico analisado em laboratórios da cidade de Salvador/BA. Destes, 28\% apresentaram colesterol total acima de $240 \mathrm{mg} / \mathrm{dL}$ (17). Sendo Salvador uma cidade com poucos imigrantes japoneses, a semelhança desses resultados salienta que os hábitos de vida ocidental atuam de modo semelhante para o surgimento de hipercolesterolemia em populações de etnias diversas. Essa hipótese é reforçada pelo estudo MESA (12), no qual 30\% dos participantes tinham concentração de LDL-C acima de seu alvo proposto pelo NCEP - ATP III (6), com pequena variabilidade entre as etnias estudadas.

Comparando nossos resultados com os obtidos no Japão (7), observa-se que os valores médios de colesterol total e LDL-C são discretamente mais elevados nos nipo-brasileiros (colesterol total $=215$ vs. $201 \mathrm{mg} / \mathrm{dL}$ e LDL-C = 130 vs. 118 mg/dL). Ressalta-se que apenas um quinto da população nipo-brasileira apresenta LDL-C $<100 \mathrm{mg} / \mathrm{dL}$, valor considerado ideal quando existe elevado risco cardiovascular (6). Uma pesquisa conduzida com imigrantes japoneses e seus descendentes na cidade de São Paulo/SP detectou maior prevalência de hipercolesterolemia em niseis, indivíduos mais aculturados ao estilo de vida brasileiro, quando comparados aos seus pais nascidos no Japão (18). Essa situação parece refletir o maior consumo de gordura e 
proteína animal pelos niseis de São Paulo/SP fato relatado em um estudo prévio (19).

Outro ponto de interesse é a presença de níveis de LDL-C mais elevados nas mulheres nipo-brasileiras, em concordância com os resultados obtidos na população de Salvador/BA (17) e do município de Cotia/SP (13). Apesar de essa diferença apontar para pior perfil de risco no sexo feminino, como já referido, a razão colesterol total/HDL e os níveis de TGs foram maiores nos homens, sugerindo um quadro metabólico mais grave no sexo masculino.

Por fim, a média da apoB no presente estudo está dentro da faixa de normalidade e não difere entre os sexos. Em outra análise feita por nosso grupo, a apoB não se associou com a presença de doença macrovascular (3). Nossos achados não valorizam a apoB como um bom marcador de risco cardiovascular nesta população nipobrasileira, com elevada prevalência de DM2. No entanto, o INTERHEART, estudando populações de diferentes etnias em todo o mundo, mas com menores prevalências de DM2, detectou que a razão apoB/ apoAl e o tabagismo foram responsáveis por dois terços do risco atribuível populacional de infarto agudo do miocárdio (20).

As correlações encontradas entre idade e colesterol total, LDL-C e HDL-C foram significativas, porém fracas, e não se observou aumento nos níveis de TG ou apoB com o avançar da idade. Assim sendo, outros fatores devem estar envolvidos na deterioração do perfil lipídico desses indivíduos em faixas etárias mais jovens, salientando-se os hábitos de vida ocidentais que foram mais assimilados pela segunda geração e a alta prevalência de DM2.

Concluímos que a hipertrigliceridemia é a anormalidade do perfil lipídico mais comum nos nipo-brasileiros, possivelmente explicada pela elevada prevalência de DM2 nessa população e talvez pelo consumo de álcool, marcadamente maior no sexo masculino (16). Os homens exibem pior perfil lipídico que as mulheres; no entanto, a idade parece ter pouca influência nesse cenário. É provável que a inadequação alimentar e o sedentarismo exerçam papel mais relevante na deterioração da saúde desses indivíduos $(11,15,16)$. A também elevada prevalência de hipercolesterolemia agrava o risco cardiovascular já conferido pela SM. Sendo assim, chamamos a atenção para a necessidade urgente de intervenção nos hábitos de vida, instituindo-se medidas preventivas e terapêuticas para atenuar o risco cardiovascular dessa população.

\section{AGRADECIMENTOS}

Esta pesquisa teve o apoio da Fundação de Amparo à Pesquisa do Estado de São Paulo (Fapesp).

\section{REFERÊNCIAS}

1. Gimeno SGA, Ferreira SRG, Franco LJ, Hirai AT, Matsumura L, Moises RS. Prevalence and 7-year incidence of type II diabetes mellitus in a Japanese-Brazilian population: an alarming public health problem. Diabetologia. 2002; 45:1635-8.

2. Fujimoto WY, Bergstrom RW, Boyko EJ, Kinyoun JL, Leonetti DL, Newell-Morris LL, et al. Diabetes and diabetes risk factors in second- and third-generation Japanese-Americans in Seattle, Washington. Diabetes Res Clin Pract. 1994; 24(Suppl):S43-52.

3. Siqueira AFA, Gimeno SGA, Abdalla DSP, Matsumura LK, Barros Jr N, Ferreira SRG. Macrovascular disease in a JapaneseBrazilian population of high prevalence of metabolic syndrome: associations with classical and non-classical risk factors. Atherosclerosis. 2007. [No prelo].

4. Siqueira AFA, Abdalla DSP, Ferreira SRG. LDL: from metabolic syndrome to instability of the atherosclerotic plaque. Arq Bras Endocrinol Metabol. 2006; 50:334-43.

5. Alberti KG, Zimmet P, Shaw J. Metabolic syndrome - a new world-wide definition. A consensus statement from the International Diabetes Federation. Diabet Med. 2006; 23:469-80.

6. Executive Summary of the Third Report of the National Cholesterol Education Program (NCEP). Expert panel on detection, evaluation, and treatment of high blood cholesterol in adults (Adult Treatment Panel III). JAMA. 2001; 285:2486-97.

7. Arai H, Yamamoto A, Matsuzawa Y, Saito Y, Yamada N, Oikawa $S$, et al. Serum lipid survey and its recent trend in the general Japanese population in 2000. J Atheroscler Thromb. 2005; 12(2):98-106.

8. Centro de Estudos Nipo-Brasileiros. Pesquisa da população de descendentes de japoneses residentes no Brasil. São Paulo: Centro de Estudos Nipo-Brasileiros, 1988.

9. King $\mathrm{H}$, Aubert RE, Herman WH. Global burden of diabetes, 1995-2025. Prevalence, numerical estimates and projections. Diabetes Care. 1998; 21:1414-31.

10. Rosenbaum P, Gimeno SG, Sanudo A, Franco LJ, Ferreira SRG. Analysis of criteria for metabolic syndrome in a population-based study of Japanese-Brazilians. Diabetes Obes Metab. 2005; 7:352-9.

11. Sartorelli DS, Freire RD, Ferreira SRG, Cardoso MA for the JB DSG. Dietary fiber and glucose tolerance in Japanese-Brazilians. Diabetes Care. 2005; 28(9):2240-2.

12. Goff DC, Bertoni AG, Kramer H, Bonds D, Blumenthal RS, Tsai $M Y$, et al. Dyslipidemia prevalence, treatment and control in the Multi-Ethnic Study of Atherosclerosis (MESA). Circulation. 2006; 113:647-56.

13. Martins IS, Marucci MFN, Cervato AM, Okani ET, Mazzilli RN, Casajus MI. Doenças cardiovasculares ateroscleróticas, dislipidemias, hipertensão, obesidade e diabetes melito em população da área metropolitana da região Sudeste do Brasil: II - Dislipidemias. Rev Saúde Pública. 1996; 30(1):75-84.

14. Lerario DDG, Gimeno SG, Franco LJ. Excesso de peso e gordura abdominal para a síndrome metabólica em nipo-brasileiros. Rev Saúde Pública. 2002; 36(1):4-11. 
15. Doro AR, Gimeno SGA, Hirai AT, Franco LJ, Ferreira SRG for the JBDSG. Análise da associação da atividade física à síndrome metabólica em estudo populacional de nipo-brasileiros. Arq Bras Endocrinol Metab. 2006; 50(6):1066-74.

16. Freire RD, Cardoso MA, Shinzato AR, Ferreira SRG for the JBDSG. Nutritional status of Japanese-Brazilian subjects: comparison across gender and generation. $\mathrm{Br} J$ Nutr. 2003; 89:705-12.

17. Lessa I, Conceição JL, Souza ML, Oliveira V, Carneiro J, Melo $\mathrm{J}$, et al. Prevalência de dislipidemias em adultos da demanda laboratorial de Salvador, Brasil. Arq Bras Cardiol. 1997; 69(6):395-400.

18. Amato RV, Cesar LA, Padua Mansur A, Hueb WA, Martins JR, Brito Viana $C$, et al. Coronary heart disease clinical manifestation and risk factors in Japanese immigrants and their descendents in the city of Sao Paulo. Arq Bras Cardiol. 2003; 81(3):229-38.

19. Cardoso MA, Hamada GS, Souza JM. Dietary patterns in Japanese migrants to southestern Brazil and their descendants. J Epidemiol. 1997; 7:198-24.

20. Yusuf S, Hawken S, Ounpuu, Dans T, Avezum A, Lanas FS, et al. Effect of potentially modifiable risk factors associated with myocardial infarction in 52 countries (the INTERHEART Study): case-control study. Lancet. 2004; 364:937-52.

\section{APÊNDICE}

\section{Membros do Japanese-Brazilian Diabetes Study Group - JBDSG}

Alcides Hirai, Amelia T Hirai, Helena Harima, Katsumi Osiro, Mario Kikuchi, Suely GA Gimeno (Departa- mento de Medicina Preventiva, Universidade Federal de São Paulo); Vania D'Almeida (Departamento de Pediatria, Laboratório de Genética, Universidade Federal de São Paulo); Laercio J Franco (Departamento de Medicina Preventiva e Social Faculdade de Medicina de Ribeirão Preto, Universidade de São Paulo); Luiza K Matsumura, Regina CS Moises (Disciplina de Endocrinologia, Departamento de Medicina, Universidade Federal de São Paulo); Marly A Cardoso (Departamento de Nutrição, Faculdade de Saúde Pública, Universidade de São Paulo); Newton de Barros Jr (Departamento de Cirurgia, Universidade Federal de São Paulo); Nilce Tomita (Faculdade de Odontologia de Bauru, Universidade de São Paulo); Katsunori Wakisaka (Japanese-Brazilian Study Center, São Paulo); Rita Chaim (Faculdade de Nutrição, Universidade Sagrado Coração de Jesus de Bauru); Sandra RG Ferreira (Departamento de Nutrição, Faculdade de Saúde Pública, Universidade de São Paulo).

Endereço para correspondência:

Antonela Ferro Antunes de Siqueira

Rua Cristiano Viana, 647, ap. 62

05411-001 São Paulo, SP

E-mail: antonela74@terra.com.br 